\title{
Carotid Artery Stenosis Correlation with Hyperhomocysteinemia in Stroke Patient Group: a Prospective Study
}

\author{
Viktorija Kenina*, Zanda Priede*, Pauls Auce**, Normunds Suna**, Andrejs Millers* \\ *Riga Stradins University, Department of Neurology, Riga, Latvia \\ **Pauls Stardins Clinical University Hospital, Department of Neurology, Riga, Latvia
}

\begin{abstract}
Summary
Introduction. Stroke is the second most common cause of death worldwide and one of the major causes of long-term disability. Carotid artery stenosis is an independent risk factor for ischemic stroke and related forms of atherosclerotic vascular disease.

Aim of the Study was to examine plasma homocysteine (tHcy) levels in the stroke patient's group with significant carotid artery stenosis, to determine hyperhomocysteinemia correlation with degree of carotid artery stenosis.

Materials and methods. This study was prospective and all patients $(n=102)$ included in the study were hospitalized in Pauls Stradins Clinical University hospital in Clinic of Neurology with diagnosis of acute ischemic stroke. In the group of significant carotidal stenosis we included 48 patients with various degree of stenosis ranging from $50 \%$ to total occlusion. Evaluations of stenosis of extracranial carotid arteries were done by duplex ultrasonography method. The blood of these patients was tested for homocysteine level by ELISA (IMMULITE 2000).

Results. Study did not demonstrated statistically significant difference between levels of tHcy in all groups. Mean homocysteine level was not significantly higher in the symptomatic carotid stenosis patient's group. Also there were no significant differences between levels of homocysteine in patient group with different degree of stenosis.
\end{abstract}

Conclusions. We found no meaningful association between a high tHcy level and extent of carotid stenosis.

Key words: hyperhomocysteinemia, stroke, carotid stenosis.

\section{INTRODUCTION}

Stroke is the second most common cause of death worldwide and one of the major causes of long term disability, furthermore mortality from stroke in Latvia compared to neighboring countries is one of the highest in the region(16). One can divide stroke into further subtypes to apply slightly different preventive strategies, for example anticoagulation or surgical carotidal endartectomy. Large artery atherosclerosis is one of the most common causes of stroke and in particular group of younger patient it is also the most frequent one (5). Raised levels of homocystein have been previously described as an independent risk factor of coronary artery disease (3) and stroke (19) and there has been already reported particular association between high homcysteine levels and ischemic stroke due to large artery atherosclerosis (4). Link between hyperhomocysteinemia and external carotid artery has already been previously investigated. Selhub J, et al have demonstrated association between raised level of homocysteine and risk of extracranial carotid artery stenosis in elderly (17), these findings have later been confirmed by several other studies (10), (18)in addition, link to severe stenosis also has been demonstrated (14), but at the same time study from Iran failed to demonstrate this association in their local population (12). Interestingly, several studies have also failed to demonstrate association of early re-stenosis following carotid endartectomy and increased level of homocysteine (2), (7).

\section{AIM OF THE STUDY}

The aim of our study was to examine plasma homocysteine (tHcy) levels in the stroke patient's group with significant carotid artery stenosis, to determine hyperhomocysteiemia correlation with degree of carotid artery stenosis.

\section{MATERIALS AND METHODS}

This research was conducted as a prospective study, and was approved by the hospital's Ethics Commission. All the research subjects $(n=48)$ were acute ischemic stroke patients admitted to the Pauls Stradins Clinical University Hospital in time period from October, 2008 till May, 2009. Stroke was defined according to WHO definition: as a focal (or at times global) neurological impairment of sudden onset, and lasting more than 24 hours (or leading to death), and of presumed vascular origin. Inclusion criteria for the study were first - ever stroke or recurring cerebral infarction with carotid artery stenosis of $50 \%$ or more; while patients with lacunar strokes, past medical history significant for oncologic or chronic inflammatory diseases, severe impairment of renal function were excluded from the study. Patients in this study were not stratified according to TOAST criteria as a goal for study was to evaluate relationship between degree of stenosis and homocysteine. All patients were separated into two groups according to grade of their stenosis - one group from $50 \%$ to $69 \%$ and those with above $70 \%$. Degree of stenosis was measured 
using flow velocity analysis with Philips 33i duplex ultrasound system. For detection of level of tHcy we used IMMULITE 2000 test system, what is chemiluminescent immunoassay highly specific for homocysteine. It is used for quantitative detection of $L$ - homocysteine. Testing system consists of two cycles in first release of bound tHcy and its conversion into $S$-Adenosyl-L-homocysteine with following immunoassay using highly specific antibody. In this study we considered result $15 \mu \mathrm{mol} / \mathrm{l}$ as raised level. SPSS for Windows 16.0 was used for data processing and analysis.

\section{RESULTS}

Mean level of homocysteine in the patients group $(\mathrm{n}=48)$ was $16,73 \pm 6,8 \mathrm{mmol} / \mathrm{l}$.

From 48 patients included in study 21 (gender: 14 male, 7 females; mean age: $64,3 \pm 11,8$ years) had stenosis above $70 \%$ (critical) and 27 (gender: 26 male, 22 females; mean age: $64,3 \pm 11,8$ years) persons had stenosis between $50 \%-69 \%$ (significant).

Mean level of tHcy in patient group with carotid stenosis above $70 \%$ was $16.26 \pm 5,4 \mathrm{mmol} / \mathrm{l}$; in the group with stenosis between $50 \%-69 \%$ mean level was 17.14 $\pm 8,06 \mathrm{mmol} / \mathrm{l}(\mathrm{p}=0.18)$.

Hyperhomocysteinemia was detected in 11 patients out of 21 in group with critical stenosis; as well as in 15 patients out of 27 in other group with significant stenosis from $50 \%-69 \%,(p=0.83)$.

\section{DISCUSSION}

In this study we did not demonstrated any statistically significant difference in level of tHcy in blood between stroke patients with critical (above $70 \%$ ) and significant $(50 \%-69 \%)$ carotidal stenosis. Apart from size of this study there is some other weakness of our study - for example we did not recorded nutritional status of included patients (for example vitamin B12 or B6 supplementation) and also we lacked proper control group matched by age and gender without cerebrovascular disease. Lack of statistically significant difference between groups also could be explained with the fact that homocysteine plays a role in atherosclerosis and stroke but not directly in determination of extent of stenosis. Link between raised level of homocysteine and other marker of atherosclerosis and vascular risk factor - Carotidal Intimal Media Tickness (C-IMT) is also controversial still, there are studies that have demonstrated link between raised level of tHcy and C - IMT (11), (8), but interestingly, some studies have demonstrated weaker link with C - IMT (6) or even absence of it(13),(9). Trials of lowering tHcy levels also have failed to show benefit in reduction of vascular events despite lowering homocysteine levels (1), in addition trial that was looking at post stroke patients and effect of lowering homocysteine failed to demonstrate improvement of C - IMT (15). Those controversies might not go against a role of homocysteine in stroke but actually just show that stroke and atherosclerosis is multi factorial illness and later might be a longstanding progradient process with more than one risk and causal factor.

\section{CONCLUSIONS}

In our study we did not demonstrate statistically significant difference in levels of blood homocysteine between patient group with critical carotid stenosis (above $70 \%$ ) and significant (50 \% - $69 \%$ ). It demonstrates that there is need for further trials in this field to continue to search for amendable risk factor in development of critical carotidal stenosis.

\section{Conflict of interest: None}

Acknowledgment: this work was supported by grant from ESF.

\section{REFERENCES}

1. Albert, C.M., et al., Effect of Folic Acid and B Vitamins on Risk of Cardiovascular Events and Total Mortality Among Women at High Risk for Cardiovascular Disease: A Randomized Trial // JAMA, 2008; 299(17): 2027-2036

2. Assadian, A., et al., Homocysteine and early restenosis after carotid eversion endarterectomy // Eur J Vasc Endovasc Surg, 2007; 33: p. 144 - 148

3. Christen, W.G., et al., Blood Levels of Homocysteine and Increased Risks of Cardiovascular Disease: Causal or Casual? // Arch Intern Med 2000; 160(4): 422-434

4. Eikelboom, J.W., et al., Association Between High Homocyst(e)ine and Ischemic Stroke due to Largeand Small-Artery Disease but Not Other Etiologic Subtypes of Ischemic Strok // Stroke, 2000; 31(5): 1069-1075

5. Grau, A.J., et al., Risk Factors, Outcome, and Treatment in Subtypes of Ischemic Stroke: The German Stroke Data Bank // Stroke, 2001; 32(11): 2559-2566

6. Held, C., et al., Correlations between plasma homocysteine and folate concentrations and carotid atherosclerosis in high-risk individuals: baseline data from the Homocysteine and Atherosclerosis Reduction Trial (HART) // Vascular Medicine, 2008; 13(4): 245-253

7. Hillenbrand, R., et al., Hyperhomocysteinemia and recurrent carotid stenosis // BMC Cardiovascular Disorders, 2008. 8(1): 1

8. Kazmierski, R., et al., Association of atherosclerotic risk factors with carotid adventitial thickness assessed by ultrasonography // Journal of Clinical Ultrasound, 2009; 37(6):333-341

9. Linnebank, M., et al., Homocysteine and Carotid Intima-Media Thickness in a German Population: Lack of Clinical Relevance // Stroke, 2006; 37(11): 2840-2842

10. Lupattelli, G., et al., Hyperhomocyst(e)inemia Is Associated with Carotid Atherosclerosis // Angiology, 1999; 50(10): 823-830

11. Malinow, M., et al., Carotid artery intimal-medial wall thickening and plasma homocyst(e)ine in asymptomatic adults. The Atherosclerosis Risk in Communities Study // Circulation, 1993; 87(4): 1107-1113 
12. Mousavi, S., M. Ghasemi, and T. Hoseini, Association between plasma homocysteine concentrations and extracranial carotid stenosis // Ann Saudi Med, 2006; 26(2): 120-122

13. Ntaios G, S.C., Hatzitolios A, Ekonomou I, Destanis E, Chryssogonidis I, Chatzinikolaou A, Pidonia I, Karamitsos D., Homocysteine and carotid intimamedia thickness in ischemic stroke patients are not correlated // Neuropsychiatr Dis Treat, 2008; 4(2): 477-9

14. Pitoulias GA, T.M., Tsiaousis PZ, Papadimitriou DK. Hyperhomocysteinemia and hypercoagulable state in carotid plaque evolution. Novel risk factors or coincidental risk predictors? // Int Angiol, 2007; 26(3): $270-8$

15. Potter, K., et al., The effect of long-term homocysteine-lowering on carotid intima-media thickness and flow-mediated vasodilation in stroke patients: a randomized controlled trial and metaanalysis // BMC Cardiovascular Disorders, 2008; $8(1): 24$

16. Sarti, C., et al. International Trends in Mortality From Stroke, 1968 to 1994 // Stroke, 2000; 31(7): 1588-1601
17. Selhub, J., et al., Association between Plasma Homocysteine Concentrations and Extracranial Carotid-Artery Stenosis // New England Journal of Medicine, 1995; 332(5): 286-291

18. Wang, H., et al., Serum level of homocysteine is correlated to carotid artery atherosclerosis in Chinese with ischemic stroke // Neurological Research. 28: 25-30

19. Homocysteine Studies Collaboration, Homocysteine and Risk of Ischemic Heart Disease and Stroke: A Meta-analysis // JAMA, 2002; 288(16): 2015-2022

\section{Address:}

Viktorija Kenina

Clinic of Neurology

Pauls Stradins University Hospital

Pilsonu iela 13

Riga, LV-1002

Latvia

E-mail: vikakenina@inbox.lv 